\title{
Trend Following Algorithms for Technical Trading in Stock Market
}

\author{
Simon Fong, Jackie Tai, Yain Whar Si \\ Faculty of Science and Technology \\ University of Macau \\ Macau SAR \\ Email: ccfong@umac.mo
}

\begin{abstract}
Trend following (TF) is a rule-based trading mechanism that taps on the movements of long-term market trend instead of relying on any forecast or external information to decide when to buy and when to sell a stock. Its simple operation is in contrast to complicated prediction methods which typically would try to predict a future trend by analyzing the historical data and may be other factors. TF makes no prediction and it is well known for its simplicity. Although TF is a popular strategy in finance and was implemented in some commercial trading system decades ago, there have not been many studies of TF in computer science. Hence the objective of this paper is to develop a computer simulator in which TF is implemented as a variety of algorithms. Through the algorithms, readers will see how different parameters are chosen, and how these TF strategies perform in a simulation with real-life data. The simulation results show that $\mathrm{TF}$ algorithm can gain an average profit of $75.63 \%$ of return-of-investment monthly. However, we observed that TF degrades in performance in proportion to the amount of fluctuation of the market trend. This finding is important to the design of technical trading systems. It implies that the fluctuation of market trend should be monitored; when it exceeds a certain threshold the TF trading should be paused to prevent loss.
\end{abstract}

Index Terms-Trend Following, Technical Trading, Java Simulator

\section{INTRODUCTION}

For many years, speculators exploit all the trading strategies in an attempt to predict stock market prices for reaping profits. Efforts of complicated analysis ranging from soft computing to experts' advice, have been exhausted to predict every market turn and momentum, in order to foresee an accurate stock market outcome. Stock market prediction is continuously being attempted. But unfortunately until now, there isn't a $100 \%$ accurate technique created to do so yet. Generally there have been two different schools of beliefs, one advocates that stock market can be predicted as the trends undergo certain patterns, the accuracy of the prediction is a matter of choosing the right prediction method and configuring the right set of parameters; the other opposite voice however supposes that stock market trend is a result of purely random movements. It is extremely difficult or almost impossible for any mathematical formula to predict the next move probabilistically with an acceptable accuracy.
By assuming that market trend is just a random variable, one simple method has been proposed called Trend Following (TF). Instead of doing any prediction, it simply rides on the trend and generates signals of buy or sell by following the long-term moves. Traders who use this approach can use current market price calculation, moving averages and channel breakouts to determine the general direction of the market and to generate trade signals. While TF is widely applied in currency markets with a good track record for major currency pairs [1], it is unconvincing that if TF can be applied in stock market though some commercial software claimed that it works.

The aim of this paper is to study in depth of TF and two variants of the TF algorithms are evaluated in terms of performance via a simulation study. The paper is structure as follow: Section 2 briefs on the two common approaches in studying stock market. One of the two popular analyses is Technical Analysis that is described in Section 3. The principles of trend following method are introduced in Section 4. The trend following methods then are formulated into computational algorithms and they are presented in Section 5, namely Static P\&Q and Adaptive $P \& Q$. Section 6 is the simulation study and discussion of the results, followed by a conclusion.

\section{BACKGROUND ON STOCK MARKET ANALYSIS}

Generally two approaches have been widely adopted for harvesting information that is used to guide stock market trading. Fundamental analysis is one of them and also the most commonly used approach in studying the markets. This analysis considers many external factors that are supposed to affect the supply and demand of a particular market. Sources from which the information can be obtained for fundamental analysis may include government policies, domestic and foreign events, political and economic news, and cooperates' annual reports. By carefully examining the supply and demand factors, or "fundamentals" for a particular market, it is believed to possibly predict changes in market conditions. These changes are then supposedly related to the fluctuation of the price of the market. It was however argued that nobody can be absolutely certain about the accuracy of the market information. Market trends change upon a flux of news that come by every day is too complex to be accurately analyzed [2]. There are too 
many factors that are composite in nature, and nobody can tell precisely the relative importance of their interrelations that collectively lead to the final outcome. One classical example failure that drove the dip of stock market years ago is the bubble burst of dot-com stocks, promoted by the hype of "new digital economy". Furious debates still continue on the efficacy of fundamental analysis, so are other analysis.

The second popular approach into market forecasting is called technical analysis that works in opposite principle of the fundamental analysis. The underlying philosophy for this approach is that the market prices well reflect all known factors at all times. So the price is already a solid performance indicator as a result of the supply and demand for that particular market. It is analogous to Occam's razor that says "The simplest explanation for some phenomenon is more likely to be accurate than more complicated explanations." Therefore technical analysis evaluates solely on the market prices themselves rather than on any fundamental factor else outside the market. Traders who are solely equipped with technical analysis suppose that a careful analysis of daily price movement as well as a long term trend is all that is required to predict a price trend for their trading.

\section{TECHNICAL ANALYSIS}

For decades traders were hoping to acquire some reliable decision making tools that would assist them in market forecasting. Many such tools are available both as commercial products and research prototypes. Predicting market trends is a hot area in the academic research community using methods of soft computing. Some popular choices are Genetic Algorithms [3], Support Vector Machines [4], and Artificial Neural Networks [5, 6]. They are used to analyse past financial data as far as twenty years ago to try divining the market direction. Many research papers in the literature claim that they yield significant results of "indicators" and they offer pretty good accuracy. Still, the question of whether technical analysis works has been a topic of contention for over three decades. Can past prices forecast future performance?

On the other hand, there is an alternative type of technical analysis that neither predicts nor forecasts. This kind is entirely based on price trend. Instead of striving to predict a market direction, this strategy is to react to the market's movements whenever they occur; hence the name Trend Following (TF). TF responds meticulously to what has recently happened and what is currently happening, rather than anticipating what will happen. TF is solely based on some statistically trading rules. In [7], a simulator has attempted to program TF into trading agents which base on linear regression of direct market trends. The obvious challenge for generating maximum profits in this strategy is how to define such rules. The rules are usually derived from the traders' judgments and are subjective in nature. A main contribution of this paper is to derive some TF rules as computer algorithms and programmed them for quantitative evaluation in an automated trading simulator.

\section{PRINCIPLES OF TREND FOLLOWING METHODS}

Some principles of trend following methods are reviewed here [8]. Of what appears to be in common in the techniques surveyed in [8], the success of Trend Following strategies depends on certain underlying assumptions. The first assumption is the regular occurrence of price trends that are resulted from a variety of factors. The trends go up and down all the time in markets. Market prices are the objective data as they are the ultimate indicators reflected from the factors. The price movements are enough for making decisions in trading, and little anything else according to $\mathrm{TF}$ strategies. Individual price histories and charts can just be used as primary data for TF trading program to operate.

Secondly, the success assumes that TF trading systems can possibly garner profit from these trends. As believed by some experts, the basic trading strategy that all trend followers try to systematize is to 'cut losses' and 'let profits run.' This basic premise is that the most profit is gained when a trader is synchronized to an enduring trend. To do this, traders need to adopt a strict discipline that minimizes human behavioral bias (i.e., intuitive or "gut" feel). It does not anticipate a trend beginning or end, and acts when the trend changes.

Based on this premise, a Performance Probability Score (PPS) model [8] was proposed as a classic approach to trend following. PPS incorporates analysis of relative valuation and relative price performance to produce risk and reward expectations for individual stocks. The core of the model is a binary separation of whether long-term relative price performance is positive or negative. Price performance is used as a key indicator derived from the price trend over a reasonable length of time. Trend following is doing the same. The basic rule of PPS is to stay with a positive or negative rating until a definable change has occurred. In other words, PPS does not anticipate changes in trends. It just reacts to changes.

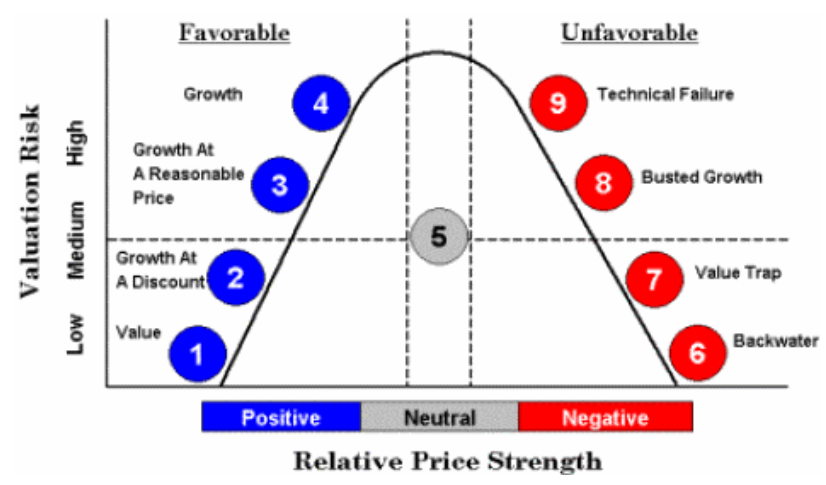

Figure 1. Division of relative price strength along the price trend. Source: Legg Mason

\section{OUR PROPOSED MODEL}

Inspired by the concept of the PPS model, our model attempts to reap profits from the difference of the initial market price and the market volatility. Changes in price mean opportunities that may lead to a gradual reduction or increase of the initial trade. Conversely, certain 
adverse price movements may lead to an exit for the whole trade. When there is a turn detected in contrary to the trend, the system will have to decide whether to signal a pre-programmed exit or wait until the turn establishes itself as a trend in the opposite direction. If the situation favours an exit as reasoned by the rules, the system will re-enter when the trend re-establishes. In order to have the TF trading system automated, we need the following elements to be considered.

Trade Management: Some rules are implemented over the decision of how much to trade over the course of the trend. This includes decision of how much to trade at each time, and how much money to risk in each trade. In order to minimize risk, for example, the trading size is reduced during periods of higher market volatility or losing periods. The trade is managed to let the profit runs when market is good, and holds back during bad times to preserve capital until more positive price trends reappear.

Market Price: The market price should be the sole input data to the system. Although there could be information derived from the market price history, as a general rule other indicators estimating where price will go next should be disregarded. In order to run the TF system in real-time, likewise must be for the availability of the latest market price. Price and time are pivotal at all times.

Rules: The main rules are simply to maximize profiles and cut losses. The rules operate systematically and objectively without consideration of any analysis of fundamental supply or demand factors. The rules automate the trading by making decisions on when to enter the market, for how long to stay in a trade for profit, and when to exit the trade if it becomes unprofitable.

We proposed a new model that is derived from PPS but with more accurate and more adaptive detection of the change(s) that trigger a buy or sell action. Hence some rules must be formulated for determining when to enter or exit a position in the market based on two important signals. The two signals that are directly derived from the market price trend tell us when to enter a market, and when to exit a market. These two signals are two fundamental variables in defining the rules of the trading model.

\section{A. Static $P$ \& Q Rules}

Trading rules are the underlying mechanism of a TF system. In our model, the rules mainly depend on two variables, namely $P$ and $Q$. $P$ is defined to be the amount of the trend in the upward direction that triggers a position to be opened. Literally $P$ stands for a proposition for signaling it is time to enter the market since certain significant progress of market growth is observed. $Q$ is the amount of opposite trend after a pivotal turn that will cause an opened position to close. $Q$ can simply be considered as a signal to quit the trade from the market in order to prevent any deep loss.

Let $T$ denotes the market trend which is a continuous curve made up of Price $(t)$ in the function of time $t$. For example in the following diagram, it will open a long position when the current Price at point $t$ over the trend $T$ advances over $P$; and it will close out the position when the trend $T$ declines deeper than $Q$.

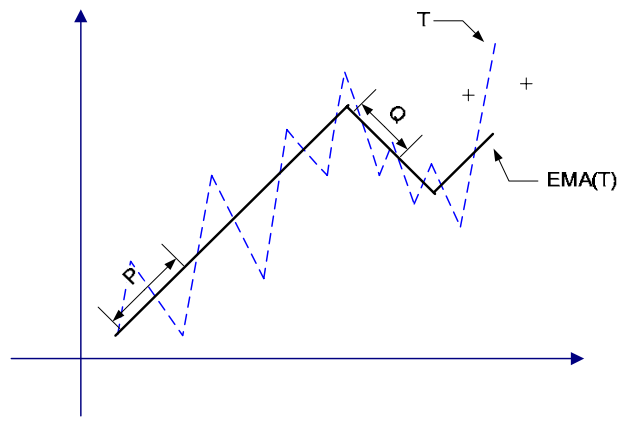

Figure 2. $\quad P$ and $Q$ over the price trend $T$ and $\operatorname{EMA}(T)$

However in reality the market price trend doesn't go constantly on a straight line; it is much volatile that the line fluctuates up's and down's repeatedly. Therefore it is not possible (at least not practical) to apply the $P \& Q$ rules directly on the trend $T$ because the frequent fluctuation will alarm off too many signals of trading actions. In order to compromise this fluctuation, an Exponential Moving Average (EMA) is adopted and upon which we applied the rules. EMA at the time of $t$ is calculated as follow,

$E M A_{(t)}=\left(\operatorname{price}_{(t)}-E M A_{(t-1)} \times \frac{2}{n+1}\right)+E M A_{(t-1)}$

where price $(t)$ is the current price at time $t, n$ is the number of periods in minute for intra-day trading or days in inter-day trading, and $t$ is any given market time. Using $P \& Q$ rules on EMA helps to smooth a fluctuating trend. The logic of the trading rule by using $P \& Q$ is shown in pseudo-code as follow.

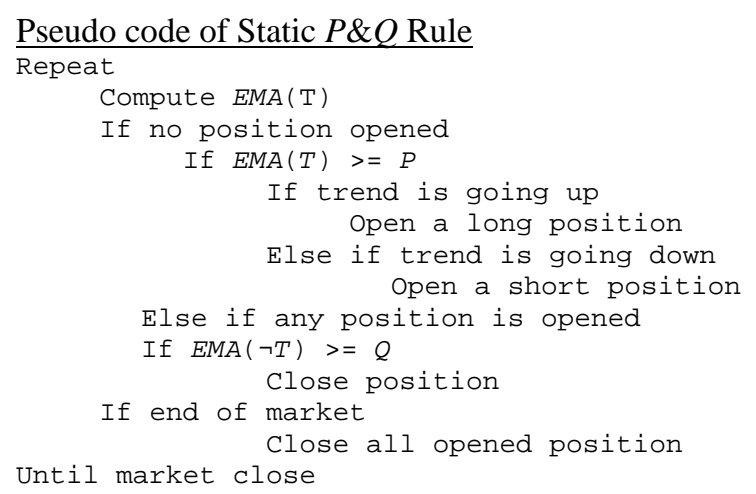

Our method as depicted in the pseudo code above, entails using a moving average, for a set of market price data, to determine when to enter the market and when to exit. Profits and losses were made along the way between buying and selling. The number of days $n$ used to calculate the moving average is found by observing which value of $n$ gave the best profit over a reasonable length of time.

In other TF systems, values for $P$ and $Q$, or such similar parameters were either chosen arbitrarily or by subjective intuition of some experienced human traders. In some cases, the two parameters are represented by a single threshold whose value was calibrated intuitively. 
In our model, the value of $P$ and $Q$ are derived empirically from the historical market price data as it is believed that market data is a reflection of the consequent trends and they are sufficient for references. The values of $P$ and $Q$, therefore can be either found by brute-force or heuristic methods based on the historical data. In brute-force method, all combinations of $P$ and $Q$ values are tried exhaustively over a sample, and observed for the best performing pair as shown in Figures 3 and 4.

Alternatively, an artificial neural network can be trained by using historical dataset to estimate the most profitable set of $P$ and $Q$ values. For instance a pair of optimal $P$ and $Q$ values could be derived from a sample of Hang Seng index future contract used in an artificial neural network as in our experiment. A forward feed neural network with a hidden layer of 20 neurons was tested to give reasonable results. A certain portion of the random sampling of dataset across ten years of records could be used as training set.

By the nature of neural network whose initial weights were set randomly and different samples were selectively used in the training phase, a range of $P$ and $Q$ values could be possibly obtained. Some post-processing was required to filter the choices of $P$ and $Q$ values into a reasonable range. If any of the $P$ and $Q$ values gets too small, then there will be too many order executions. If the value is too large, for example 1000 , there may be no order execution at all.

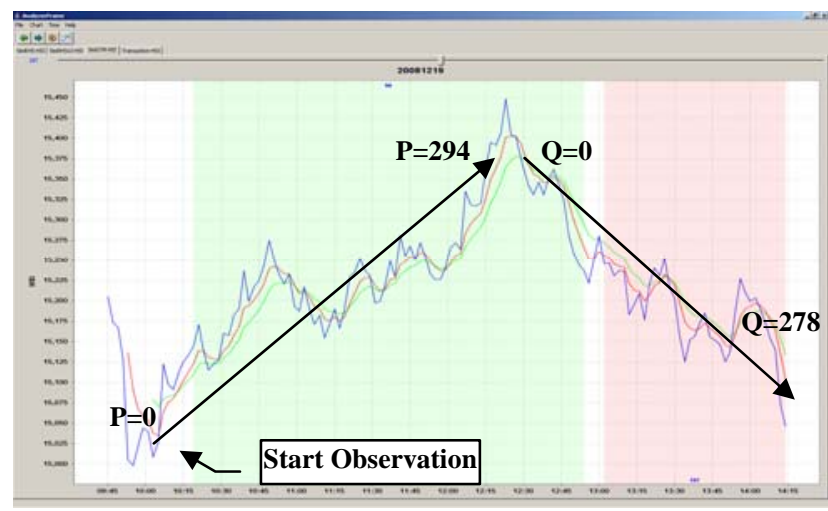

Figure 3. Ranges of $P$ and $Q$ values obtained from heuristic measures

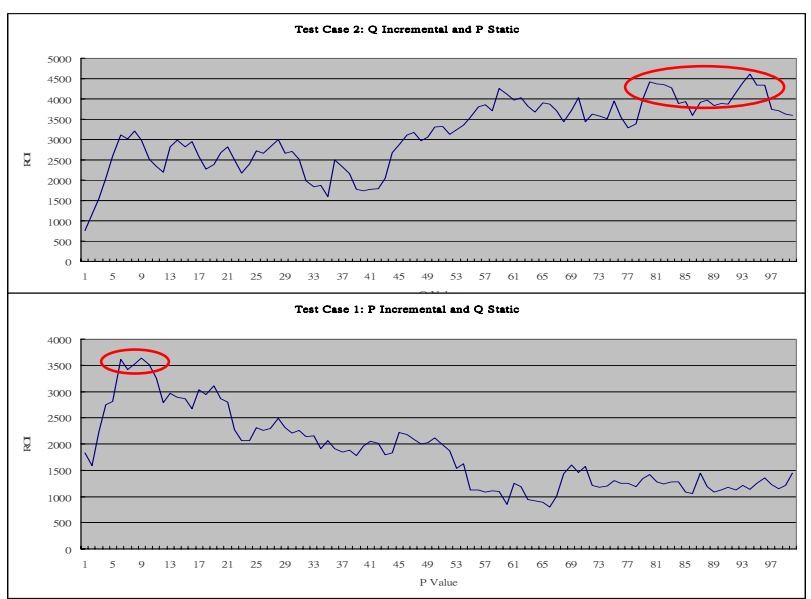

Figure 4. By holding the other value constant, it is possible to have multiple values of $P$ and $Q$ that generate good profits

\section{B. Adaptive $P \& Q$ Rules}

Previously, the values of $P$ and $Q$ are obtained from considering the combo values that give the best performance over a relative long period of time. It is intuitively observed that the lengths of $P$ and $Q$ that worked well in a particular section of a trend may not do the same in other situations. Hence the process of generating the $P$ and $Q$ values should be modified such that they would dynamically change to adapt to the realtime market trend.

In this adaptive operation, the amounts of progress and decline that govern the buying and selling are calculated in real-time at regular intervals along the market trend. Hence, $P^{\prime}$ and $Q^{\prime}$ are two new variables that are used in the trend following algorithm for the same purposes. They have basically the same meaning as $P$ and $Q$ in the rules of trading except that their values may change dynamically in different parts of the trend instead of remaining constants

In this case, some additional technical analysis that helps determining the buying and selling positions in real-time is desirable for adjusting the $P^{\prime}$ and $Q^{\prime}$. Relative Strength Index $(R S I)$ is one of the popular approaches for this purpose. RSI is a technical momentum indicator that compares the magnitude of recent gains to recent losses in an attempt to determine overbought and oversold conditions of an asset. The advantage is that it reflects signals of overbought or oversold in real time as the market progresses. It is calculated using the following formula:

$$
\begin{aligned}
& R S I_{(t)}=100-\frac{100}{1+R S_{(t)}} \\
& R S_{(t)}=\frac{A U_{(t)}}{A D_{(t)}} \\
& A U_{(t)}=\frac{U p_{(t)}+U p_{(t-1)}+\ldots . . U p_{(t-n+1)}}{n} \\
& A D_{(t)}=\frac{\operatorname{Down}_{(t)}+\operatorname{Down}_{(t-1)}+\ldots . . . \text { Down }_{(t-n+1)}}{n},
\end{aligned}
$$

where $A U$ is average price upward in $n$ periods, $A D$ is average price downward in $n$ periods, $n$ is the number of $R S I$ periods that is usually taken as 14 by most analysts

One example is given below in Figure 5, the RSI ranges from 0 to 100 . An asset is deemed to be overbought once the RSI approaches the 70 level, meaning that it may be getting overvalued and is a good candidate for a pullback. Likewise, if the RSI approaches 30 , it is an indication that the asset may be getting oversold and therefore likely to become undervalued. According to [9], the following three observations are classical and well known by stock market technical analysts.

- Above 50, the internal strength of the market is considered bullish; below there, considered bearish.

- Above 70 is a bullish danger zone, considered to represent an overbought market that will correct sooner or later.

- Below 30 is a bearish danger zone, considered to represent an oversold market that will rally sooner or later. 
The ideas that hold true for oscillators in general hold true with the RSI. The oscillator will frequently turn around before the price does - for example, a price still rising that is accompanied by a falling RSI produces a bearish divergence between price and oscillator, a major warning that the uptrend is running out of steam.

Note that 70 and 30 are typical values for $R S I_{\max }$ and $R S I_{\text {min }}$ respectively. These two thresholds can be arbitrarily chosen by traders. In our experiments, we set $R S I_{\max }$ and $R S I_{\min }$ to be 60 and 40 that narrows the range by an offset of 10 for less-risky trading.

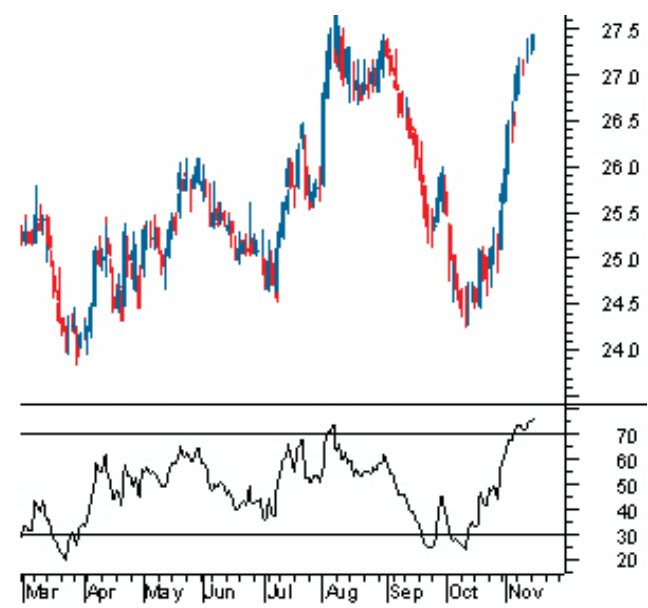

Figure 5. Illustration of RSI and its maximum and minimum thresholds (Source: Investopedia)

A trader using RSI should be aware that large surges and drops in the price of an asset will affect the RSI by creating false buying or selling signals. The RSI is best used as a valuable complement to other stock-picking tools. In our adaptive $P \& Q$ strategy, $R S I$ is used as a main reference index in calculating $P^{\prime}$ and $Q^{\prime}$ in real-time. By studying the interaction of how the indicator reacts to the market, we can derive the criteria as follow, which depict the situation ready for a position to open

For long position, at $P^{\prime}$

1. Price is advancing

2. $R S I(t)$ is greater than $\operatorname{EMA}(R S I(t))$

3. $\operatorname{EMA}(R S I(t))$ is less than 40 or greater then 60 For short position, at $Q^{\prime}$

1. Price is declining

2. $R S I(t)$ is less than it $\operatorname{EMA}(R S I(t))$

3. $\operatorname{EMA}(R S I(t))$ is less than 40 or greater then 60

The following diagram shows an example of a long position opened at time 10:35 after the long position criteria are met, and closed out at time $13: 36^{1}$ when the short position criteria are met. The values of $P^{\prime}$ and $Q^{\prime}$ now change adaptively and dynamically according to and along with the RSI. As the trading goes, the criteria assess the fluctuating trend of the market and trigger positions to be open or close.

${ }^{1}$ In Hong Kong stock market there's a two hours break between morning and afternoon sessions, to avoid this discontinuation on the chart, we shifted the time backward, and joined this two sessions into one, so $13: 36$ is equivalent to $15: 36$

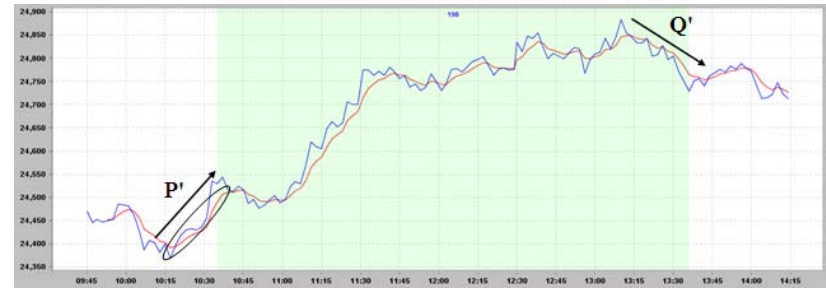

Figure 6. Figure 6. $P^{\prime}$ and $Q^{\prime}$ are determined by the criteria of adaptive strategy.

To program this strategy into an automated trading simulator, the following pseudo-code is used:

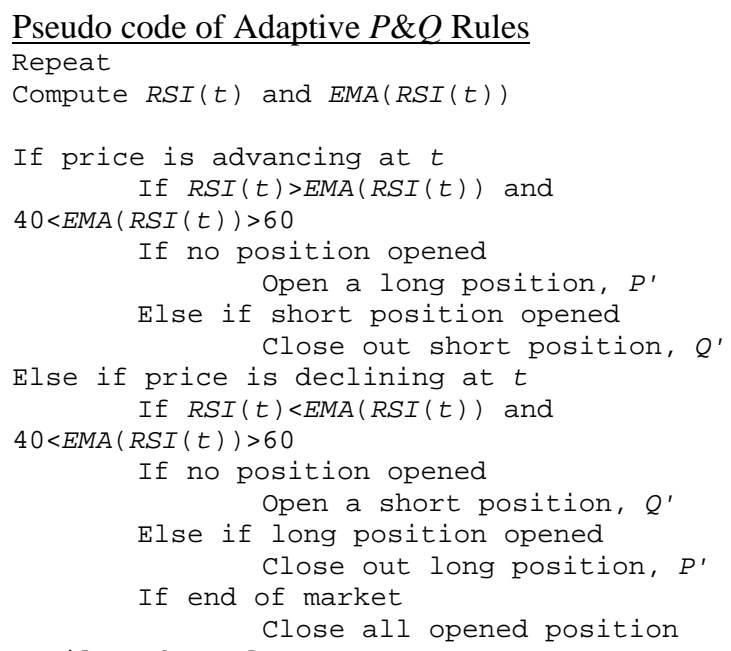

Until Market Close

\section{SIMULATION EXPERIMENTS AND RESULTS}

The purpose of this simulation experiment is to verify that the effects of $P \& Q$ strategies in applying trend following, and to compare the static and adaptive $P \& Q$ strategies. Most importantly the simulator is to prove the possibilities that TF can yield profits in market trading, by strictly following the rules in a computer program.

Our experiment simulates intraday trading on Hang Sang future contract, which is stored in data file with daily records in year 2008; with a total size of 355 days. Hang Seng Index is founded in 1969 November, it is composed of 36 different securities including some wellknown companies such as Li Ka Shing's Cheung Kong, HSBC holdings and China Mobile. It is the second largest stock market in Asia in total capital that is worth of USD $\$ 1.6$ trillion by 2007 January.

We use the indices as our to-be-estimated target to represent the average of all companies and balanced portfolios which approximates the overall market returns. The reason that we use indices rather than individual stocks is that we suppose the market index can balance the bias for choosing stocks and eliminate the influence of single stock.

One important resultant variable in our simulation is the returns on investment (ROI) on common shareholder's equity. In this study, we are going to use the average ROI of overall stock in a specified market as a performance indicator for comparing the two variants of trend following strategies. 
The ROI is calculated based on the assumption that one contract is traded with the initial capital of HKD 100,000 , the average monthly ROI is the total ROI divided by the number of months in the length of the simulation.

- Read in the stock market data from a file.

- Calculate the EMA and RSI for some given values of parameters, such as $n$ the period of time.

- Feed the data into the two $P \& Q$ strategies, static and adaptive, and generate the buying and selling signals - During the simulation, when the reversal of trend gives rise to $P$ and $Q$ conditions, then our trading strategy regard this situation as a buying signal and selling signal respectively.

- Simulate the trading by calculating the profits each day and subtract costs if there is a trade.

- Run this for many values of $n$ and summarize the results including the ROI.

The following diagram shows the simulation of both strategies with respect to the Hang Seng index performance as a base line. The base line could represent a buy-and-hold strategy that suffers an overall depreciation in value when the whole trend is sinking down in performance as shown in the diagram. The two
$P \& Q$ strategies of TF however in contrast, are gaining in increasing the values of the initial capital; the lines show that profits can be reaped even during those bad times when the market index was going down.

The dates are normalized, taking the starting date of the input data as the initial date in the simulation. Figure 8 shows a snapshot of daily profits gained and daily losses incurred on a daily basis. In general, when the total trading events are averaged out, there are more profits than losses both in magnitudes and in counts.

Table 1 shows a summary list of results extracted from the simulation graph in Figure 7. It shows in comparison of the performance of the static and adaptive versions of $P \& Q$ strategies in trend-following. Overall, we can see that the adaptive $P \& Q$ outperforms the static one because the rules of opening and closing a positions can be better estimated based on real-time RSI. They are adaptive in real-time to the ever changing market trend. Both trendfollowing strategies however are yielding impressive results in average monthly ROI, amounted to $67.67 \%$ and $75.63 \%$ respectively based on our simulation results.

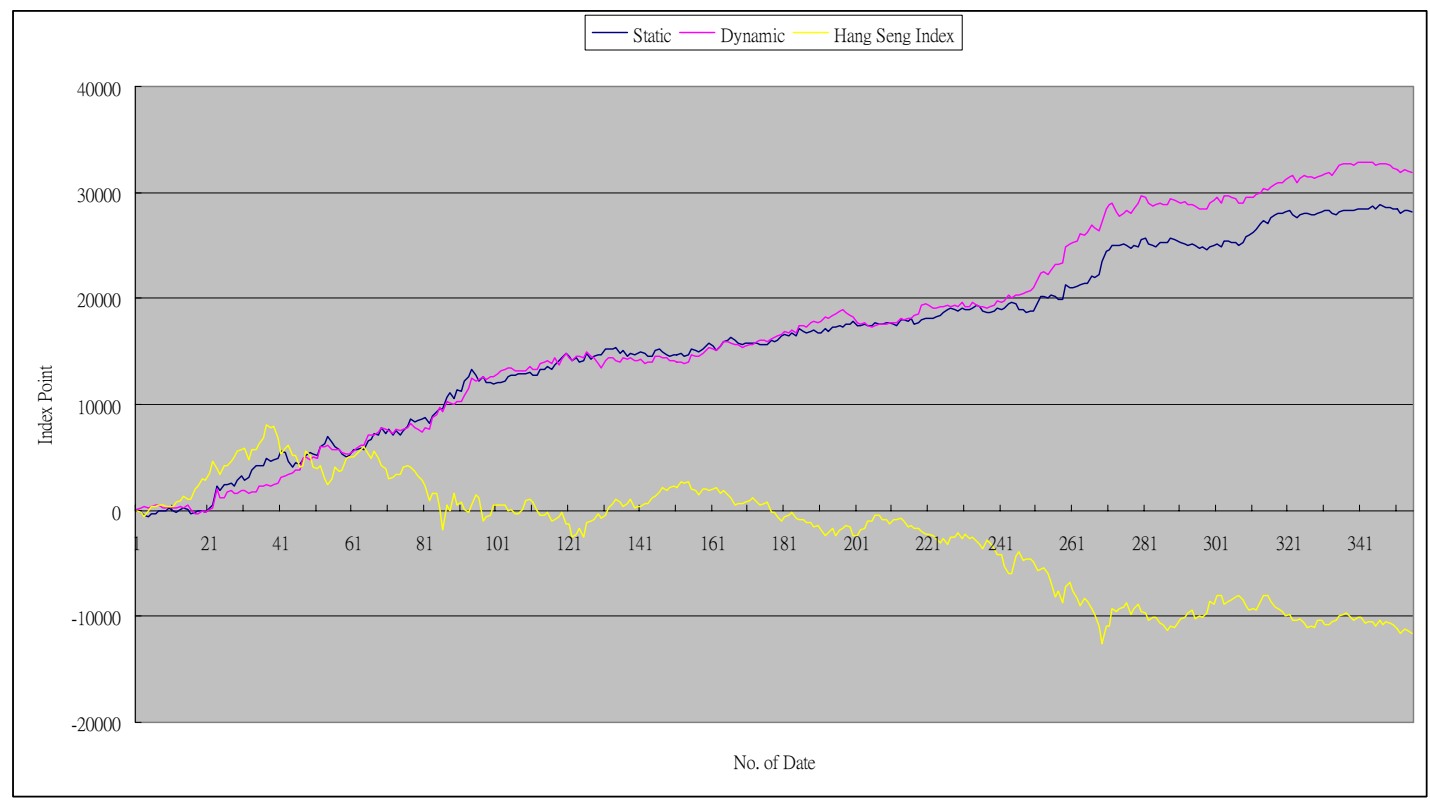

Figure 7. Simulation experiment of trading on Hang Seng Index using trend-following strategies.

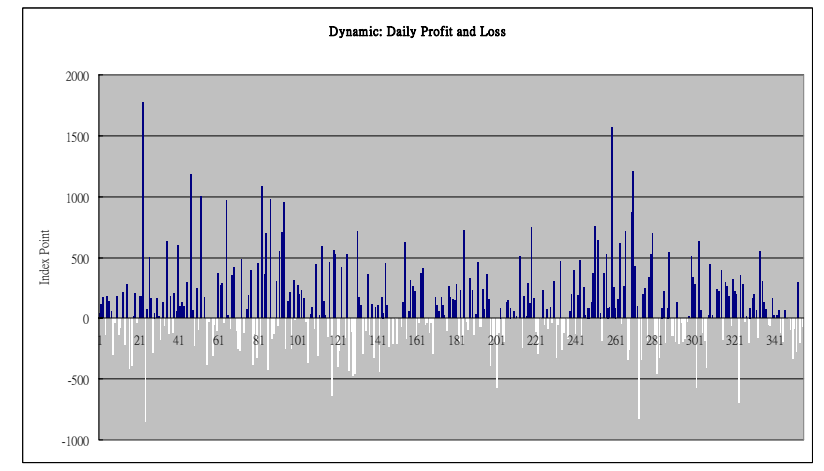

Figure 8. Daily profits and losses occurred in the adaptive P\&Q TF.
TABLE I.

SUMMARY OF RESULTS FROM THE SIMULATION EXPERIMENT

\begin{tabular}{|l|l|l|}
\hline Simulation & Static & Dynamic \\
\hline Total Index Point & 28151 & 31896 \\
\hline Net Worth (HKD) & 1407550 & 1594800 \\
\hline Total Trade & 900 & 1370 \\
\hline Cost (Commission in HKD) & 54000 & 82200 \\
\hline P\&L (Net Worth - Cost) & 1353550 & 1512600 \\
\hline Average Monthly ROI & $67.67 \%$ & $75.63 \%$ \\
\hline ROI & $1353.55 \%$ & $1512.60 .6 \%$ \\
\hline
\end{tabular}


Further analysis from Figure 8, one would be curious about, 'does it exist any method which we could eliminate or at least minimize the occurrence of daily losses?' To this end, we investigate what probably caused the losses and under what circumstances the losses happened, though the randomness of the price movement is the fundamental assumption for TF. Previous studies from $[10,11]$ found that high return is related to low market volatility by using Genetic Algorithms. Market volatility is the relative rate at which the price of a stock moves up and down. If the price of a stock moves up and down rapidly over short time periods, it has high volatility. The impact of high volatility confuses the TF algorithms that capitalized the earning from long positions of a trend.

With this clue, the scenarios of the top best and the top worst performance by the TF algorithms were examined. We discovered that the TF performance indeed is much affected by the frequency of market volatility. Snapshots of some top and worst performing samples are shown in Figure 9. It can be seen that in general, market trend that is relatively smooth yields good results. Highly fluctuating trend zigzags the signals of the TF algorithms and that leads to their bets misplaced.

In order to confirm this phenomenon, we simulated artificial fluctuations in market prices and tested the performance of the TF algorithms in terms of profits-orlosses. The perturbation of fluctuation is injected into market prices by this formula:

$$
\text { Fluctuate }(t)=\left(\operatorname{COS}_{(e)} \times C \times R_{(t)}\right)+B
$$

where

COS: is the geometry cosine which creates the basic wave structure

$e:$ is the angle that controls the fluctuation frequency

$C$ : is a constant that controls the fluctuation depth

$R$ : is a random number that creates the saw-toothed sharp

$B$ : is a base price along which the generated fluctuation price will oscillate.

With $B$ arbitrarily chosen from our simulation input data, levels of fluctuation in different intensity can be artificially generated in a controlled manner. Some levels of fluctuation in market data are shown in Figure 10 and the corresponding results of the trade by the TF are listed in Table 2.

In this example case, we can see from the results when the fluctuation level reaches $45 \%$, the automated trading system starts to lose money. Different value of $B$ has been chosen and the experiment was repeated many times. The values of Profit or Loss may take slightly different values but out of all the trials the same phenomenon was observed - loss was resulted as the fluctuation of the market prices hits $45 \%$.

This finding has a profound significance. An automated trader system can practically monitor the current fluctuation of the market price that could be calculated by the same way of market volatility. When the fluctuation exceeds a pre-calculated threshold (e.g. $45 \%$ in our experiment), the TF may temporarily pull out from the trade until the fluctuation settles down again.
Since different stock market whose data may induce a different threshold, it is suggested to conduct a precalculation of the fluctuation threshold by running the TF algorithm with that set of data.

TABLE II.

PERFORMANCE OF TF ALGORITHM IN DIFFERENT FLUCTUATIONS

\begin{tabular}{|c|c|c|}
\hline Fluctuation (\%) & Profit or Loss & No. of trades / day \\
\hline 0 & 0 & 0 \\
\hline 5 & 356 & 1 \\
\hline 10 & 240 & 1 \\
\hline 15 & 426 & 2 \\
\hline 20 & 380 & 4 \\
\hline 25 & 363 & 3 \\
\hline 30 & 494 & 3 \\
\hline 35 & 100 & 3 \\
\hline 40 & 44 & 4 \\
\hline 45 & -194 & 4 \\
\hline 50 & -561 & 4 \\
\hline 55 & -170 & 5 \\
\hline 60 & -701 & 5 \\
\hline 65 & -1104 & 6 \\
\hline 70 & -1371 & 6 \\
\hline 75 & -1840 & 6 \\
\hline 80 & -1063 & 6 \\
\hline 85 & -2557 & 6 \\
\hline 90 & -1783 & 7 \\
\hline 95 & -2907 & 7 \\
\hline
\end{tabular}

CONCLUSION

Technical trading can be either predictive or reactive. One type of technical trading is called Trend Following (TF). TF works by just following the trend, they don't predict any future trend. TF is usually represented by trading rules that are programmed into a trading system. The rules are meticulously executed at times of losses and wins. The rules are rational and human emotions are eliminated. In the case of trading in stock market, TF automates the buying or selling process depending on the position of the price relative to a long time moving average value. In this paper, we programmed TF in two algorithms and verified their performance via a computer simulator and real-life stock market data. The contribution of this work forms a cornerstone for future development of automated trading system based on TF principles which are discussed and evaluated. Two different TF strategies namely static P\&Q and adaptive P\&Q are proposed and our simulation results show that they give positive trading profits even when the stock market index is declining at the bad times. Furthermore, the situations where profits and losses occurred were investigated from our log files. It was observed that TF results in good profit when the market volatility is low, and vice versa. Therefore the experiments were repeated with market data injected with different levels of artificially generated fluctuation ranging from zero to $100 \%$. By going through the performance we found a critical level of fluctuation over which TF starts to yield a loss. Thus TF system is suggested to keep track of the market fluctuation, pause the trading if the critical point of fluctuation is reached to prevent loss. 


\section{REFERENCES}

[1] J. James, Simple trend-following strategies in currency trading Quantitative Finance 3, 2003, C75-C77

[2] C. Robertson, S. Geva, R. Wolff, "What types of events provide the strongest evidence that the stock market is affected by company specific news", Proceedings of the fifth Australasian conference on Data mining and analystics, Vol. 61, Sydney, Australia, 2006, pp.145-153.

[3] D. Fuente, A. Garrido, J. Laviada, A. Gomez, "Genetic algorithms to optimise the time to make stock market investment”, Genetic and Evolutionary Computation Conference, Seattle, USA, July 2006, pp.1857-1858

[4] Y. Sai, Z. Yuan, K. Gao, "Mining Stock Market Tendency by RS-Based Support Vector Machines", The 2007 IEEE International Conference on Granular Computing, Silicon Valley, USA, November 2007, pp.659-664

[5] S. Xu, B. Li, Y. Shao, "Neural Network Approach Based on Agent to Predict Stock Performance", IEEE International Conference on Computer Science and Software Engineering, Vol.1, Wuhan, China, December 2008, pp.1223-1225

[6] R. Makwana, Financial Forecasting Using Neural Networks, ADIT Journal of Engineering, Vol.2, No.1, December 2005, pp.56-59

[7] A. Sherstov and P. Stone, "Three Automated StockTrading Agents: A Comparative Study", In P. Faratin and J.A. Rodriguez-Aguilar, editors, Agent Mediated Electronic Commerce VI: Theories for and Engineering of Distributed Mechanisms and Systems (AMEC 2004), Lecture Notes in Artificial Intelligence, Springer Verlag, Berlin, 2005, pp. 173-187

[8] Covel, Michael, Trend Following: How Great Traders Make Millions in Up or Down Markets, Financial Times, Prentice Hall Books, 2004

[9] J. Welles Wilder, New Concepts in Technical Trading Systems, 1978

[10] F. Allena, R. Karjalainenb, "Using genetic algorithms to find technical trading rules", Journal of Financial Economics, Vol. 51, Issue 2, February 1999, pp.245-271

[11] P. Stephanos, G. Stephanides, "Improving Technical Trading Systems by using a new MATLAB-based Genetic Algorithm Procedure", Mathematical and Computer Modelling, Vol.46. 2007, pp.189-197

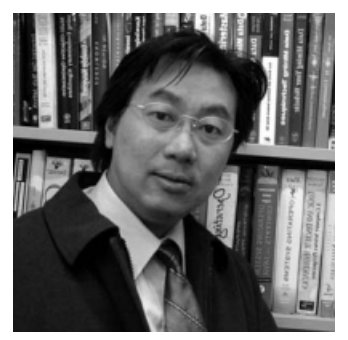

Simon Fong graduated from La Trobe University, Australia, with a 1st Class Honours BEng. Computer Systems degree and a $\mathrm{PhD}$. Computer Science degree in 1993 and 1998 respectively. Simon is now working as an Assistant Professor at the Computer and Information Science Department of the University of Macau. He is also one of the founding members of the Data Analytics and Collaborative Computing Research Group in the Faculty of Science and Technology. Prior to joining the University of Macau, he worked as an Assistant Professor in the School of Computer Engineering, Nanyang Technological University, Singapore. Before his academic career, Simon took up various managerial and technical posts, such as systems engineer, senior IT consultant and e-commerce director in Melbourne, Hong Kong and Singapore. Some companies that he worked include Hong Kong Telecom, Singapore Network Services, AES Pro-Data Australia and United Oversea Bank, Singapore. Dr. Fong has published over 120 peer-referred international conference and journal papers, mostly in the areas of E-Commerce and Data-mining.

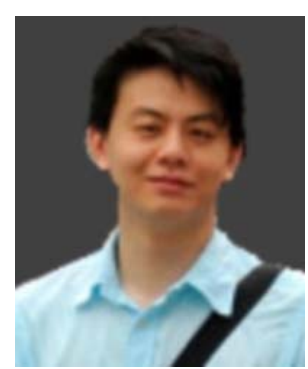

Jackie Tai is now studying a Master programme, major in E-Commerce Technology, at the University of Macau. Jackie has been working as an engineer in the I.T. department of Direcção dos Serviços de Finanças, Macau.

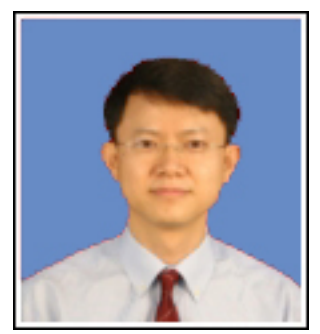

Yain-Whar $\mathbf{S i}$ is an Assistant Professor at the University of Macau. $\mathrm{He}$ obtained a $\mathrm{PhD}$ degree in Information Technology from Queensland University of Technology, Australia in year 2005. In year 1997 and 1994, he graduated with a MSc degree and BSc degree in Software Engineering respectively from University of Macau. His research interests are in the areas of business process management and decision support systems. 

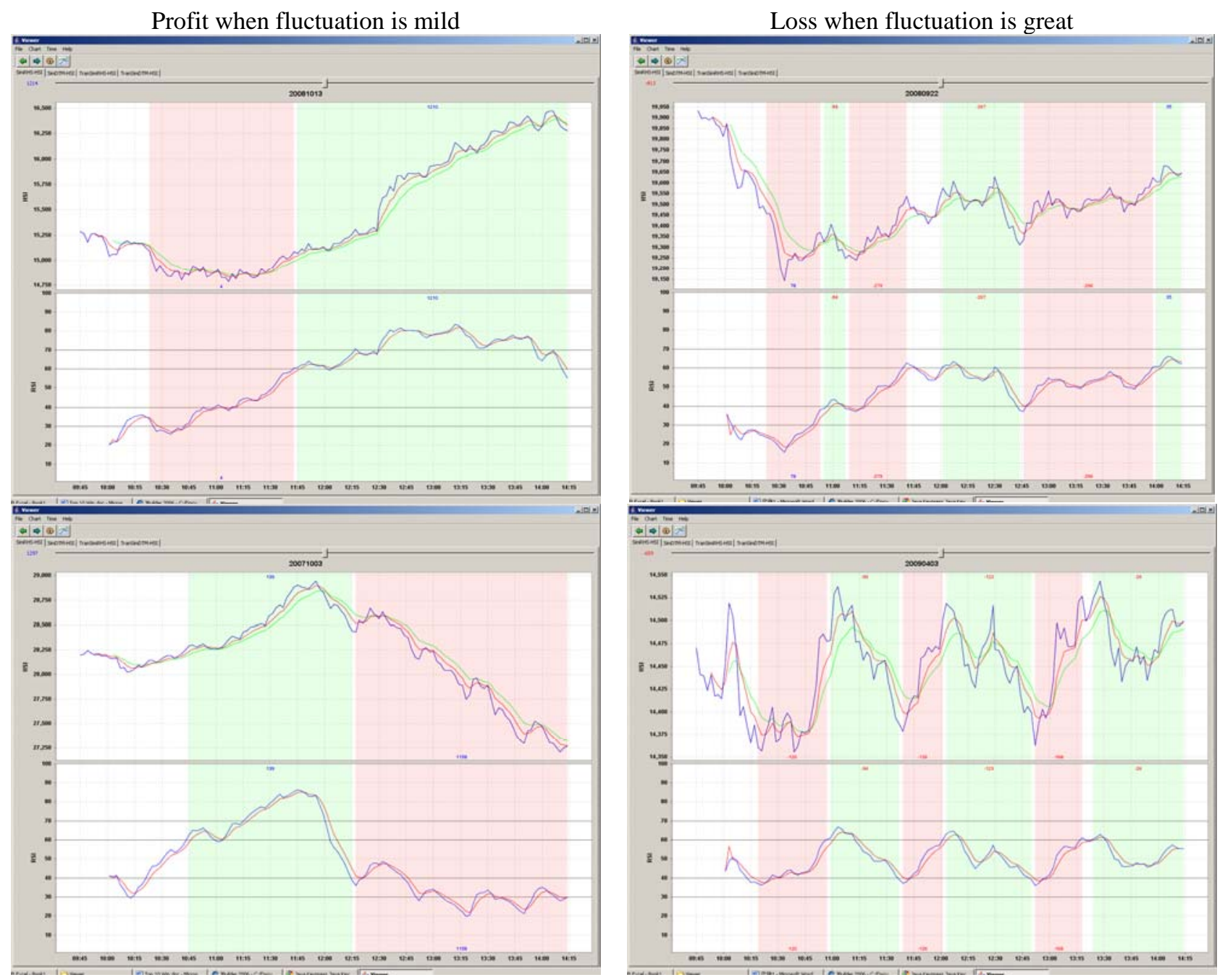

Figure 9. Samples of TF runs that made the most profit and most loss 

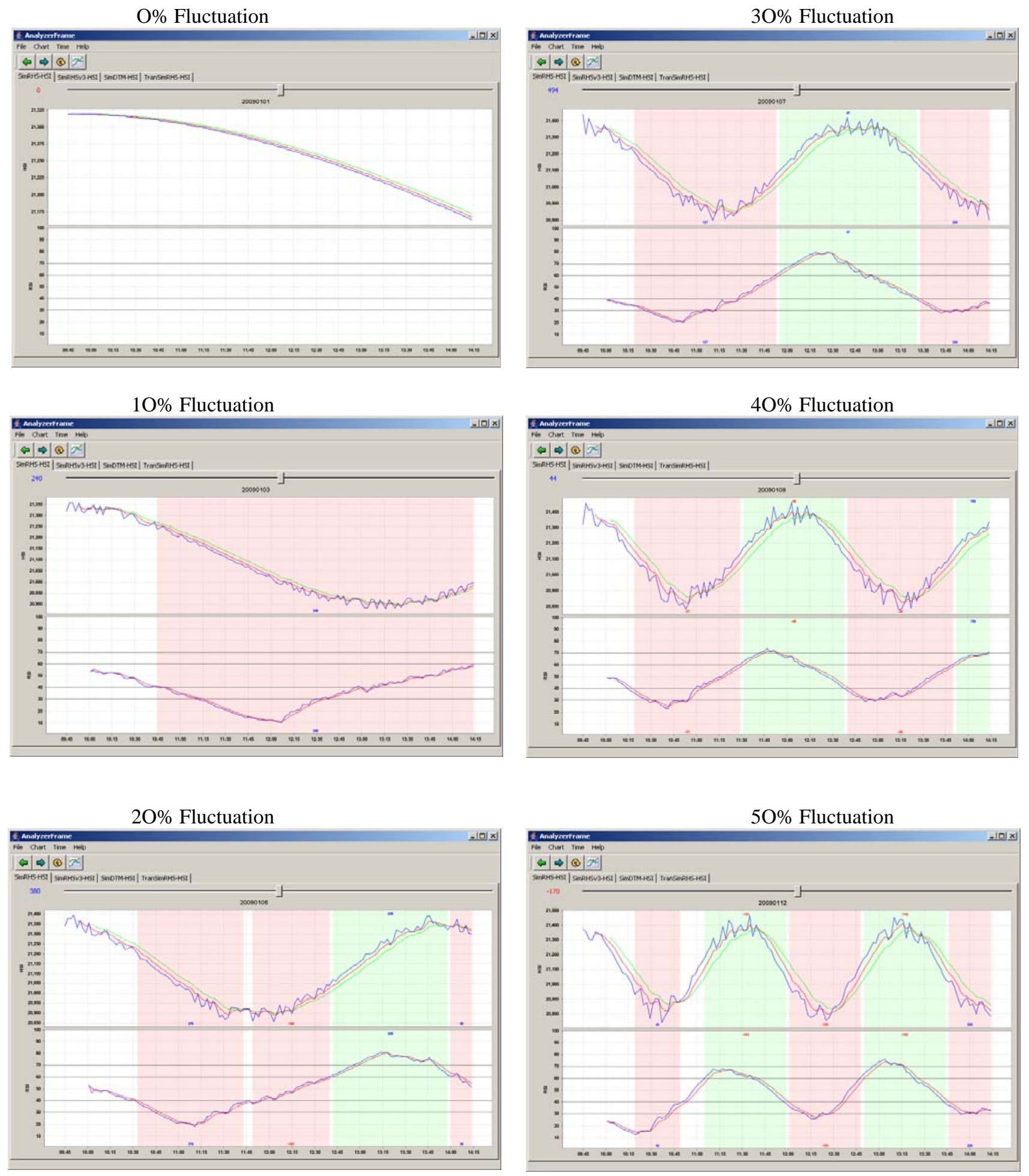

Figure 10. Market data that are manipulated with different level of fluctuation 